\title{
A CHARACTERIZATION OF THE ALGEBRA OF HOLOMORPHIC FUNCTIONS ON A SIMPLY CONNECTED DOMAIN
}

\author{
DERMING WANG and SALEEM WATSON \\ Department of Mathematics and Computer Science \\ California State University, Long Beach \\ Long Beach, California 90840 \\ (Received August 3, 1987)
}

ABSTRACT: Let A be a singly-generated F-algebra. It is shown that A is isomorphic to $H(\Omega)$ where $\Omega$ is a simply connected domain in $C$ if and only if $A$ has no topological divisors of zero. It follows from this that there are exactly three F-algebras (up to 1somorphism) which are singly generated and have no topological divisors of zero.

KEY WORDS AND PHRASES. F-algebras, holomorphlc functions, topological divisors of zero 1980 MATHEMATICS SUBJECT CLASSIFICATION CODE. $46 J 35$

\section{INTRODUCTION.}

The algebra $H(\Omega)$ of holomorphic functions on a domain $\Omega \subset \mathbb{C}$ with pointwise operations and compact-open topology is an interesting example of an F-algebra. This algebra has been characterized in terms of some of the special properties it enjoys that are derived from the fact that it consists of holomorphic functions. (See for example [1], [2], [3], [4] and [5] for characterizations in terms of the local maximum modulus principle, the Cauchy estimate, Montel's theorem, the existence of derivations, and Taylor's theorem.) In [6] a characterization of the algebra of entire functions in terms of Liouville's theorem is given.

Watson [5] shows that an F-algebra A which has a Schauder basis that is generated by an element $z \in A$ with open spectrum is algebraically and topologically isomorphic to $H(\Omega)$ where $\Omega$ is an open disk in $\mathbb{C}$. In this paper we study $F$-algebras that are generated by a single element $z$ (without requiring that $z$ generate a basis for $A$ ). Of course, this condition alone is not enough to completely describe the algebra $H(\Omega)$ among $F$-algebras. We will show, however, that this together with the condition that A has no topological divisors of zero, completely characterizes $H(\Omega)$ for a simply connected domain $\Omega$. It follows from this that there are exactly three singly generated $\mathcal{F}$-algebras (up to isomorphism) which have no topological divisors of zero.

\section{PRELIMINARIES.}

An $F$-algebra is a complete metrizable locally m-convex algebra. (All the algebras we consider are assumed to be commutative algebras over $\mathbb{C}$.) The topology of such an algebra is given by an increasing sequence of seminorms $\left\{p_{n} \mid n \in \mathbb{N}\right\}$. Each $p_{n}$ determines a Banach algebra $A_{n}$ which is the completion of $\mathrm{A} / \operatorname{ker}\left(p_{n}\right)$. If $\mathrm{n} \leq \mathrm{m}$ then the natural homomorphism 
from $\mathrm{A} / k e r\left(p_{m}\right)$ to $\mathrm{A} / \operatorname{ker}\left(p_{n}\right)$ induces a norm decreasing homomorphism $\pi n m: A_{m} \rightarrow A_{n}$ whose range is a dense subalgebra of $A_{n}$. The Banach algebras $A_{n}$ with maps $\pi_{n m}$ form an inverse limit system and $\lim \left(A_{n}, \pi_{n m}\right)$ is topologically and algebraically isomorphic to $A$.

The maximal ideal space of $A$ is the space . $M(A)$ consisting of all non-zero contınuous multiplicative linear functionals on A endowed with the Gelfand topology. This topology is the weak topology on $\mathcal{H}(\mathrm{A})$ generated by the Gelfand transforms $\hat{x}: \mathcal{N}(\mathrm{A}) \rightarrow \mathbb{C}$ defined by $x(f)=f(x)$. The map $\gamma: A \rightarrow \hat{A}$ is a continuous homomorphism onto the algebra $\hat{A} \Xi C(\mathcal{N}(A))$ of Gelfand transforms. For each $n \in \mathbb{N}$ the quotient map $\pi_{n}$ from $A$ onto $A / k e r\left(p_{n}\right)$ induces a homeomorphism $\pi_{n}^{*}$ of the maximal ideal space $n\left(A_{n}\right)$ of $A_{n}$ onto a compact subset $M_{n}$ of $\cdots(A)$. For $n \leq m$ we have $M_{n} \subset M_{m}$ and . $N(A)=U M_{n}$.

The spectrum of $z \in A$ is the set $\sigma=\sigma(z)=\{f(z) \mid f \in . M(A)\}$. For each $n \in N$ the set $\sigma_{n}=\sigma_{n}(z)=\left\{f(z) \mid f \in M_{n}\right\}$ and $\sigma=\cup \sigma_{n}$. The element $z \in A$ generates $A$ if $A$ is the smallest closed subalgebra containing $z$ and $e$ (the identity of $A$ ). In this case the spectrum map $\varphi: M(A) \rightarrow \sigma(z)$ defined by $f \mapsto f(z)$ is a continuous bijection [7].

An element $z$ in a Banach algebra $B$ is a topological divisor of zero if the multiplication map $M_{z}: A \rightarrow z A$ is not an isomorphism (i.e., does not have a continuous inverse). In an $F-$ algebra $\mathrm{A}, \boldsymbol{z}$ is called a topological divisor of zero if for each sequence $\left\{p_{n}: n \in \mathbb{N}\right\}$ of seminorms defining the topology of $A$ there exists $k \in N$ such that $\pi_{k}(x)$ is a topological divisor of zero in the Banach algebra $A_{k}[8$, pp. 46-47].

3. CHARACTERIZING $H(\Omega)$.

Let $\Omega \subset \mathbb{C}$ be a simply connected domain. The algebra $H(\Omega)$ of holomorphic functions on $\Omega$ is an $\mathcal{F}$-algebra in the compact-open topology. It is well known that $H(\Omega)$ has no (nonzero) topological divisors of zero [9], and is singly-generated. We will show that these last two

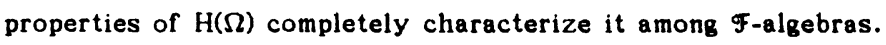

For the rest of this paper $A$ will denote an $F$-algebra with identity $e$ which is generated by $z$, where $z$ is not a scalar multiple of $e$, and which has no nonzero topological divisors of zero.

LEMMA 1. A is semisimple and so the Gelfand transform is a bijection.

PROOF: Suppose $y \in \operatorname{Rad}(A), y \neq 0$. Then $\sigma(y)-\{0\}$ and by [8, Propositon 11.8] $y$ is a topological divisor of zero.

LEMMA 2. The spectrum $\sigma(x)$ is a domain in $\mathbb{C}$.

PROOF: If $\lambda \in \sigma(z)$ is a boundary point of $\sigma(z)$, then again by [8, Proposition 11.8], z- $\lambda e$ is a topological divisor of zero. Thus $\sigma(z)$ is open.

If $\sigma(z)$ includes the two components $U_{1}$ and $U_{2}$, then the characteristic functions $h_{1}$ of $U_{1}$ and $h_{2}$ of $U_{2}$ are analytic on $\sigma(z)$. By the functional calculus there exist $x_{1}, x_{2} \in A$ with $\hat{x}_{1}=h_{1}(\hat{z})$ and $\hat{x}_{2}=h_{2}(\hat{z})$. Clearly $\hat{x}_{1} \hat{x}_{2}=0$ so by Lemma $1 x_{1} x_{2}=0$ and thus these elements are nonzero (topological) divisors of zero.

LEMMA 3. The domain $\sigma(z)$ is simply connected.

PROOF: Let $\varphi: \mathcal{M}(A) \rightarrow \sigma(z)$ be the spectrum map and for $t \xi \sigma(z)$ we use the notation $f_{t}=\varphi^{-1}(t)$. For each $x \in A$ define $\tilde{x}: \sigma(z) \rightarrow \mathbb{C}$ by $\tilde{x}(t)=\hat{x}\left(\varphi^{-1}(t)\right)=x\left(f_{t}\right)$.

We show that $\tilde{x}$ is analytic on $\sigma(z)$. Since $A$ is generated by $z$ there exists a sequence of polynomials $p_{n}=p_{n}(z)$ converging to $x$ in $A$ and so $f\left(p_{n}(z)\right) \rightarrow f(x)$, for every $f \in \mathcal{M}(A)$. For $t \in \sigma(z), \quad p_{n}(t)=p_{n}\left[\left(f_{t}(z)\right]=f_{t}\left[p_{n}(z)\right]\right.$ converges to $f_{t}(x)=\tilde{x}\left(f_{t}\right)=\tilde{x}(t)$, so each $\tilde{x}$ is a pointwise limit of polynomials on $\sigma(x)$. We now show that this convergence is uniform on 
compact subsets of $\sigma(z)$ and so each $\tilde{x}$ is analytic on this spectrum. Since A has no topological divisors of zero, for each $n: \mathbb{N}$ there exists $m n$ such that $\sigma_{n}=$ int $\sigma_{m}$ (see Arens [9]). So without loss of generality, we may assume that for $n=1,2$, ,

$$
\sigma_{n} \cdots \operatorname{lnt} \sigma_{n+1} \cdots \sigma_{n+1} \quad \text { and } \quad \sigma=\bigcup 1 n t \sigma_{n}=\bigcup \sigma_{n} \text {. }
$$

Since $\varphi \mid M_{n}$ is a homeomorphism onto its image $\varphi\left(\mathrm{M}_{n}\right)=\sigma_{n}$, it follows that if $\mathrm{K}$ is a compact subset of $\sigma$ there exists $n E \mathbb{N}$ such that $\mathrm{K}=$ int $\sigma_{n}-\sigma_{n}$. Thus $\varphi^{-1}(K) \sqsubset \varphi^{-1}\left(\sigma_{n}\right)=M_{n}$ and so $\varphi^{-\cdot}(\mathrm{K})$ is a compact subset of $\cdot n \cdot(\mathrm{A})$. Now $p_{n} \rightarrow r$ so by the continuity of the Gelfand map $\gamma$, $\hat{p}_{n} \rightarrow \hat{x}$ in $\hat{A}$, 1.e., the convergence is uniform on compact subsets of,$n(A)$. Thus for $\epsilon>0$ and sufficiently large $n$,

$$
\left|p_{n}\left(f_{t}\right)-\hat{x}\left(f_{t}\right)\right|<\epsilon
$$

for $f_{t} E \varphi^{-i}(K)$, which is the same as

$$
\left|p_{n}(t)-\tilde{x}(t)\right|<\epsilon
$$

for $t \in K$. Thus each $\tilde{x}$ is the limit of polynomials, uniformly on compact subsets of $\sigma(z)$, and hence is analytic there.

Let $h \in H(\sigma(z))$. We show that $h$ is the limit of polynomials in $H(\sigma(z))$, then it follows that $\sigma(z)$ is simply connected. Lising the functional calculus for $\mathcal{F}$-algebras we find $x \in A$ such that $\hat{x}(f)=h(\hat{z}(f)), f E_{-}(A)$. Therefore $h=\tilde{x}$. This together with the precedıng paragraph completes the proof.

LEMMA 4. $\sigma(z)$ is homeomorphic with $\mathcal{L}(\mathrm{A})$.

PROOF: The map $\varphi$ is a continuous bijection. But $\hat{x} \circ \varphi^{-1}=\tilde{l}$ is continuous for each $\hat{x} \in \hat{A}$ and so the continuity of $\varphi^{-1}$ follows from the fact that the topology of $\mathcal{M}(\mathrm{A})$ is the weak topology generated by $\hat{\mathrm{A}}$.

Lemma 4 may also be derived from [7, Theorem 1.3]. Notice that Lemmas 3 and 4 imply that $\mathcal{N}(\mathrm{A})$ is homeomorphic to the open unit disc. We now prove our main result.

THEOREM 1. An $F$-algebra $A$ is algebraically and topologically isomorphic to $H(\Omega)$ for a simply connected domain $\Omega$ if and only if $A$ is singly-generated and has no nonzero topological divisors of zero.

PROOF: That the $\mathcal{F}$-algebra $H(\Omega)$ has these properties is discussed at the beginning of this section.

Conversely, let $\tilde{\mathrm{A}}=\{\tilde{x} \mid x \in \mathrm{A}\}$ and equip $\tilde{\mathrm{A}}$ with the compact open topology. From the proof of Lemma 3, $\tilde{A}=H(\sigma(z))$ algebraically and topologically. Also, $\hat{A}$ and $\tilde{A}$ are isomorphic as $\mathcal{F}$-algebras via the map $\delta: \hat{\mathrm{A}} \rightarrow \tilde{\mathrm{A}}$ by $\hat{x} \mapsto \tilde{x}=\hat{x} \circ \varphi^{-1}$. Since the Gelfand map $\gamma: A \rightarrow \hat{\mathrm{A}}$ is bijective by Lemma 1 , it follows that the map $\delta \sigma \gamma$ is a continuous bijection of $A$ onto $\tilde{A}=H(\sigma(z))$. The open mapping theorem now yields the result.

The notion of topological divisor of zero we used above is that due to Michael [8, p. 47]. Our Theorem 1 does not remain valid if that notion is replaced by the stronger definition of Arens [10] (called strong topological divisor of zero by Michael). In fact, the $F_{-a l g e b r a}$ $C[X \rrbracket$ of formal power series (with the topology of pointwise convergence in the coefficients) is singly generated and has no strong topological divisors of zero [11]. But this algebra is not isomorphic to $H(\Omega)$ for any domain $\Omega$.

The Riemann mapping theorem yields the following corollary:

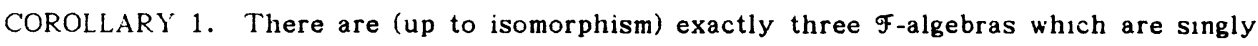
generated and have no nonzero topological divisors of zero. Namely, $\mathbb{C}$, the algebra $H(D)$ where $\mathbb{D}$ is the open unit disk, and the algebra $\&$ of entire functions. 
Birtel [6] (see also [12] and [13]) gave a characterization of the algebra of entıre functions as a singly-generated Liouville algebra without topological divisors of zero. A Liouville algebra is an $\mathcal{F}$-algebra in which every element with bounded spectrum is a scalar multiple of the identity. We give another proof of Birtel's theorem based on our Theorem 1.

THEOREM 2. (Birtel) An $F$-algebra $A$ is topologically and algebraically isomorphic to the algebra $\delta$ of entire functions if and only if $A$ is a singly-generated Liouville algebra with no nonzero topological divisors of zero.

PROOF: By Theorem $1, \sigma(z)$ is simply connected and $A$ is isomorphic to $H(\sigma(z))$. If $\sigma(z) \neq \mathbb{C}$ then there is a one-to-one analytic function $\psi$ from $\sigma(z)$ onto $D$. Thus there exists $x \in A$ such that $\hat{x}=\psi>\hat{z}$. Clearly $x$ is not a scalar multiple of $e$ and $\sigma(x)=D$, contradicting the assumption that $\mathrm{A}$ is Liouville.

A natural extension of the notion of a simply connected domain to $\mathbb{C}^{n}$ is that of a Runge domain. If $\Omega$ is a Runge domain in $\mathbb{C}^{n}$ then $H(\Omega)$ is $n$-generated and has no nonzero topological divisors of zero. We pose the question of whether a finitely-generated $F$-algebra $A$ with no nonzero topological divisors of zero is isomorphic to $H(\Omega)$ for a Runge domain $\Omega$. In the case that $A$ has a finitely-generated Schauder basis in which the joint spectrum of the generators is an open set in $\mathbb{C}^{n}$, it is shown in [14] that $A$ is isomorphic to $H(\Omega)$ for a complete logarithmically convex Reinhardt domain $\Omega$.

\section{REFERENCES}

1. RUDIN, W. Analyticity, and the maximum modulous principle, Duke Math. $\underline{\mathrm{J}} . \underline{20}$ (1953), 449-458.

2. ARENS, R. Dense inverse limit rings, Mich. Math. J. $\underline{5}$ (1958), 169-182.

3. MEYERS, W.E. Montel algebras on the plane, Canad. J. Math. 22 (1970), 116-122.

4. CARPENTER, R. Characterizing hol $(\Omega)$, Proc. Amer. Math. Soc. 53 (1975), 57-59.

5. WATSON, S. F-algebras with cyclic bases, Comment. Math. 23 (1983), 329-334.

6. BIRTEL, F.T. Singly-generated Liouville F-algebras, Mich. Math. J. $\underline{11}$ (1964), 84-94.

7. BROOKS, R.M. On the spectrum of finitely-generated locally m-convex algebras, Studia Math. 29 (1958), 143-150.

8. MICHAEL, E.A. Locally multiplicatively-convex topological algebras, Mem. Amer. Math. Soc. 11 (1952).

9. ARENS, R.F. Joint spectra in topological algebras, Conference on Analytic Functions, Inst. for Adv. Study, Vol. 2, Princeton Univ. Press, 1957.

10. ARENS, R. Linear topological division algebras, Bull. Amer. Math. Soc. $\underline{53}$ (1947), 623-630.

11. KUCZMA, M.E. On a problem of E. Michael concerning topological divisors of zero, Colloq. Math. 19 (1968), 295-299.

12. BROOKS, R.M. On singly-generated locally m-convex algebras, Duke Math. J. 37 (1970), 520-536.

13. DALY, J.T. On Liouville F-algebras, Studia Math. 40 (1971), 1-16.

14. STEWART, J., WATSON, S. Topological algebras with finitely-generated bases, Math. Annalen 271 (1985), 315-318. 


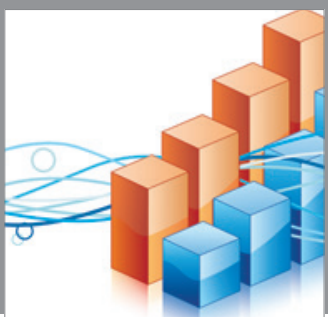

Advances in

Operations Research

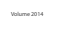

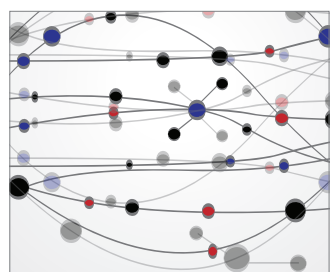

\section{The Scientific} World Journal
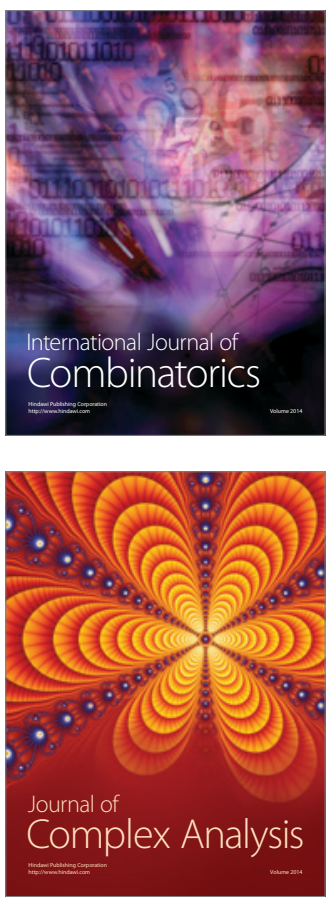

International Journal of

Mathematics and

Mathematical

Sciences
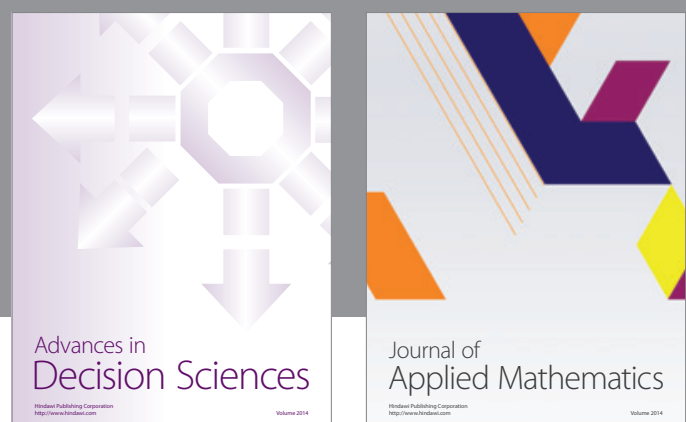

Journal of

Applied Mathematics
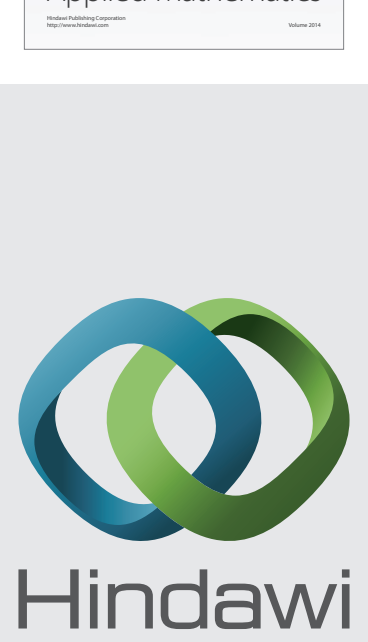

Submit your manuscripts at http://www.hindawi.com
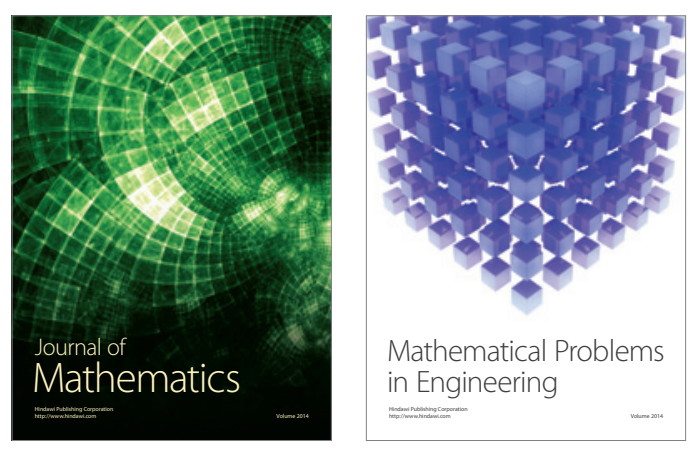

Mathematical Problems in Engineering
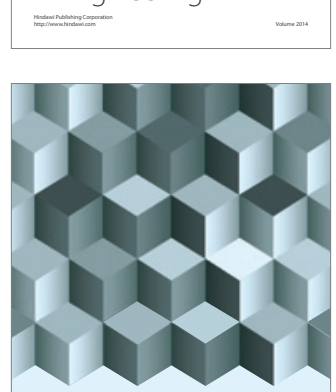

Journal of

Function Spaces
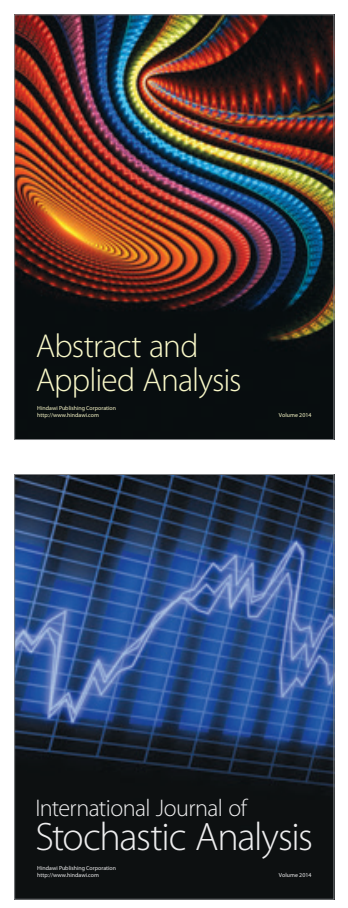

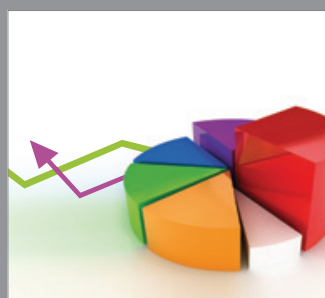

ournal of

Probability and Statistics

Promensencen
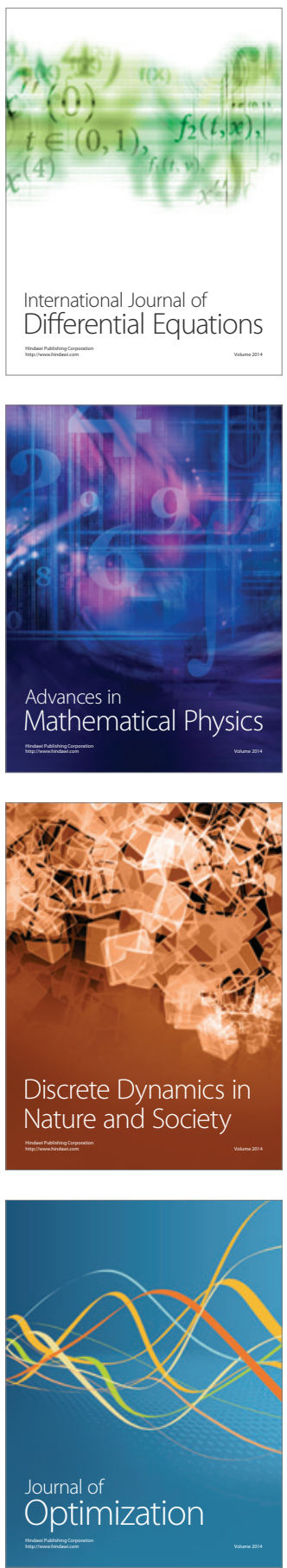